Case Report:

\title{
A Large Intrathoracic Mass in A Patient With Neurofibromatosis Type 1
}

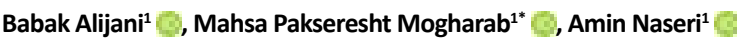

1. Department of Neurosurgery, Poursina Hospital, School of Medicine, Guilan University of Medical Sciences, Rasht, Iran

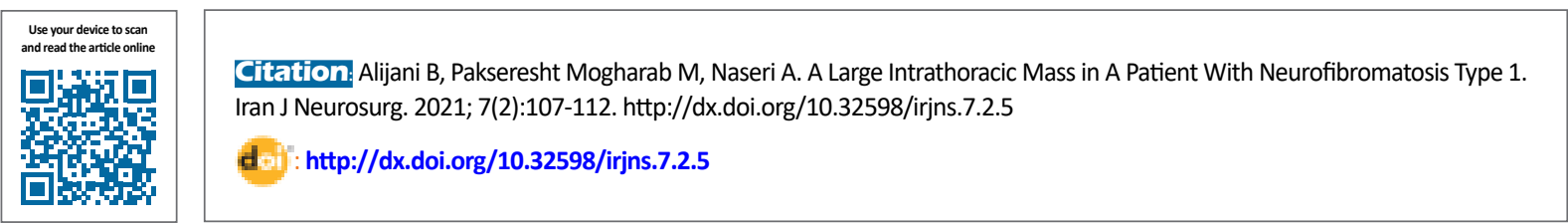

(ㄷ) 0 (8)

Article info:

Received: 23 Aug 2020

Accepted: 10 Nov 2020

Available Online: 01 Apr 2021

Keywords:

Dural ectasia, Meningocele, Neurofibromatosis, Scoliosis

\section{A B STRACT}

Background and Importance: Dural ectasia is circumferential extension or dilatation of the dural sac, it is commonly associated with Neurofibromatosis type 1 (NF1). Because NF1 is associated with a high likelihood of tumor formation, it is critical to distinguish it from other posterior mediastinal cancers such as neurofibroma, neuroblastoma, and ganglioneuroma. Scoliosis is the most frequently observed feature in NF1 patients with spinal deformities. Moreover, there are several distinctive radiographic features, including dural ectasia, defective pedicles, and spondylolisthesis, which are relatively less common in these patients. Surgery may be performed for stabilization of the spine.

Case Presentation: The present study reports on a 62-year-old female with neurofibromatosis type 1 who was referred for an unusual chest X-ray and chest Computed Tomography (CT) revealing a thoracic mass while she was hospitalized and under observation for dyspnea. The chest X-ray film showed homogenous opacity with a well-defined margin in the right apex. The chest CT showed an enlarged intervertebral foramen and defected vertebral arch around the mass and scoliosis. Although the pre-operative diagnosis was dumbbell type neurinoma, the tumor was found to be a protrusion of dura mater with spinal fluid out of the spinal canal. Part of the wall was excised, the residual opening was repaired, and surgical stabilization was performed.

Conclusion: Retrospectively, magnetic resonance imaging showed the characteristics of thoracic dural ectasia and anterior meningocele, which, in an asymptomatic case, require regular radiographic follow-ups. Surgical intervention is an alternative for patients with spinal deformities or symptomatic patients due to the adverse effects of spinal cord compression and mediastinal structures.

\section{*Corresponding Author:}




\section{Highlights}

- Dural ectasia is one of the most prevalent clinical manifestations of neurofibromatosis type 1. It should be differentiated from posterior mediastinal tumors.

- Dural ectasia may be accompanied by spinal deformities.

- Surgery may be required to stabilize the spine or when symptoms appear due to the imposed pressure on the surrounding organs.

\section{Plain Language Summary}

Dural ectasia is circumferential expansion or dilatation of the dural sac. We report a case of a 62-year-old female patient with neurofibromatosis type 1 who was referred to Poursina Hospital for an unusual mass in her chest. In addition, a spinal deformity was found at the mass level. The patient had shortness of breath and back pain. We performed the surgery on her and modified the deformity and sampled the cystic mass. The mass was found to be dural ectasia which was incidentally diagnosed.

\section{Background and Importance}

$\mathbf{N}$ eurofibromatosis Type 1 (NF1), with a prevalence of 1 in 3000, is among the most frequently observed autosomal dominant disorders [1]. The NF1 gene was discovered to be on chromosome $17 q 11.2$ [2]. Nearly $50 \%$ of NF1 cases are attributable to novel mutations [3]. Rare tumors, such as pheochromocytoma, brain tumors, and malignant peripheral nerve sheath tumors are more common in NF1. However, those tumors which frequently spread into the lung, breast, and colon, are not usually present in these patients [4]. Therefore, mass-like lesions are more likely to be malignant in NF1 patients. Spinal deformities may appear alongside NF1 being categorized into two types based on radiographic evaluation: non-dystrophic and dystrophic [5]. The former is like idiopathic scoliosis while the latter has its own distinctive features (such as transverse process spindling, vertebral wedging, and intervertebral foraminal enlargement, etc). Additionally, scoliosis is the most frequent skeletal manifestation in affected NF1 patients, with an incidence rate of $10 \%$ to $71 \%[6,7]$. Dural ectasia, spondylolisthesis, and defective pedicles are infrequently seen in NF1 patients. Therefore, awareness of these deformities contributes to an early diagnosis and optimization of the treatment [8]. The present research reports on a 62-year-old NF1 female patient with a large intrathoracic mass which was diagnosed accidentally.

\section{Case Presentation}

A 62-year-old female, a known case of NF1, was admitted to Poursina Hospital, Rasht, Iran due to an unusual chest X-ray and chest CT showing a thoracic mass, while she was hospitalized and under observation for dyspnea and a probable COVID-19 diagnosis during the coronavirus pandemic in Iran. She had back pain for two years, which was relieved by using medication. Moreover, no history of trauma was reported. However, physical examination revealed extensive café-au-lait spots, cutaneous neurofibromas, and axillary freckling which were consistent with the diagnosis of NF1. Muscle force and neurological examination were normal. The patient had a family history of neurofibromatosis as her daughter had NF1 as well. Chest radiography showed a huge mass and scoliosis (Figure 1A). A $17 \mathrm{~cm}$ homogeneous cystic mass with a thin wall in the right paravertebral area, spreading into the spinal canal via the T4-5-6 neural foramen, was revealed in chest CT (Figure 1B) and MRI (Figure 1C). To prevent the progressive deterioration of pain and spine deformity, sampling of the mass and surgical stabilization of the spine were performed (Figure 2). After one year of follow-ups, she showed no sign of dyspnea, and the back pain was reduced.

Under general anesthesia, a long-level posterior fusion from T3 to T7 using pedicle screws was performed. During the procedure, it was not possible to set the pedicle screws on the right side of T6 and the two sides of the T4-T5 pedicles. 

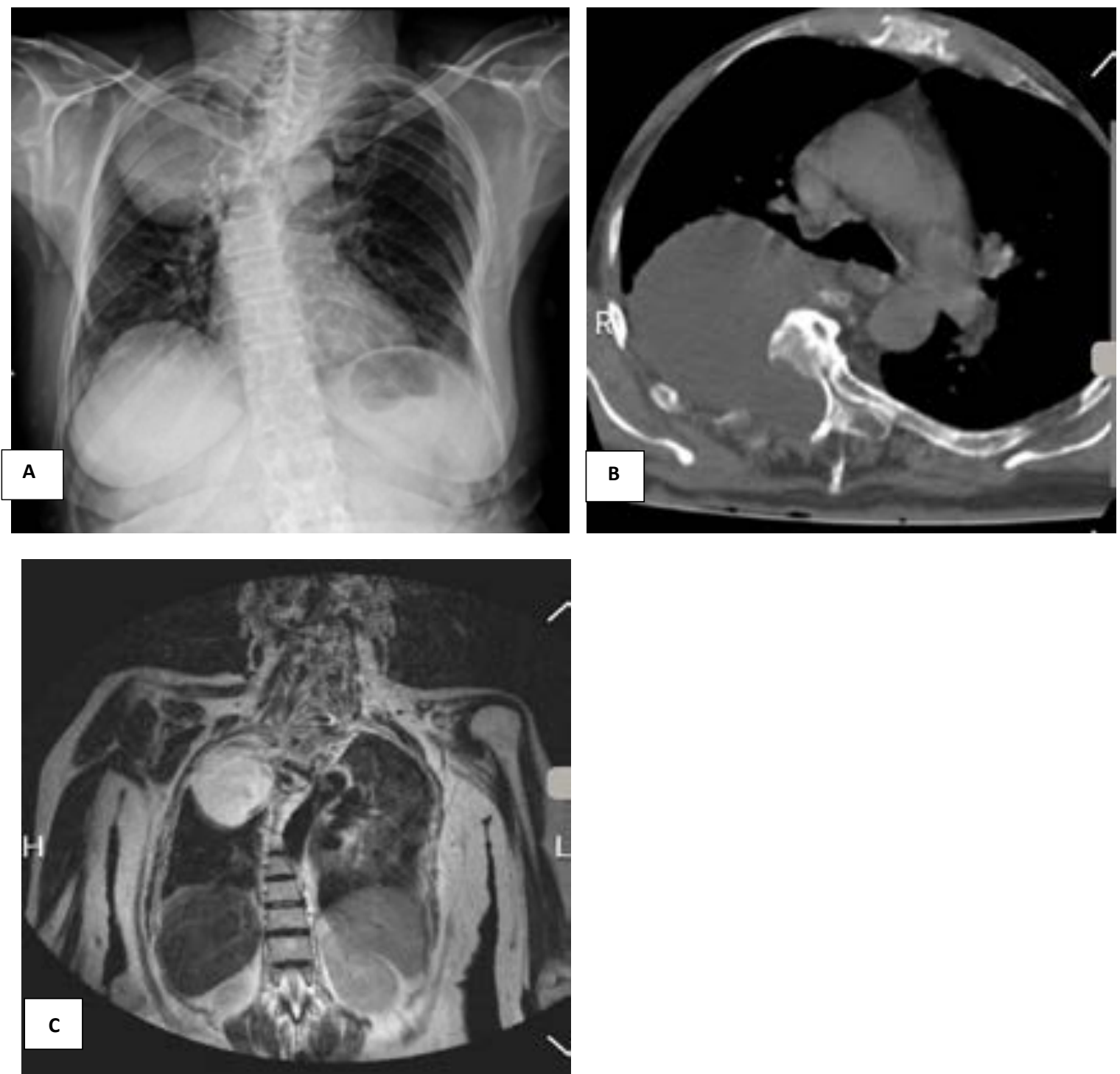

Figure 1. Pre-operative

A: Chest radiograph showing scoliosis and a large mass in the right side of thorax; B: A $17 \mathrm{~cm}$ low-attenuation mass in the left paraspinal space, eroding the right side of T4-T7 vertebrae and extending into the spinal canal through the T4-6 neural foramen on chest computed tomography; C: Coronal T2-weighted spin-echo magnetic resonance imaging of the dorsal spine indicating homogenous, cystic mass in the right paraspinal space and the neural foramen at the left T4-6 level.

\section{Discussion}

The extension of the dural sac characterizes dural ectasia which most frequently occurs in the lumbosacral spine [9]. Although dural ectasia is related to Marfan syndrome, it is usually diagnosed in patients suffering scoliosis, neurofibromatosis, ankylosing spondylitis, and Ehlers-Danlos syndrome [10]. There is a relationship between the widening of the dural sac, known as dural ectasia, and the dystrophic alteration seen in neurofibromatosis type 1 . Dural ectasia appears as meningocele through larger neuroforamina, causing bone deg- radation and organ displacement [11]. An association has been observed between meningioma and spinal nerve fibroma with neurofibromatosis type 1 that can protrude laterally and erode the neuroforamina [11]. In addition, because the thoracic region muscles are weaker and the pressure gradient of the subarachnoid space is higher than that of the thorax, lateral dural ectasia is commonly seen in this region. Moreover, it is essential to differentiate dural ectasia from other tumors, such as neuroblastoma, neurofibroma, and ganglioneuroma [12]. 


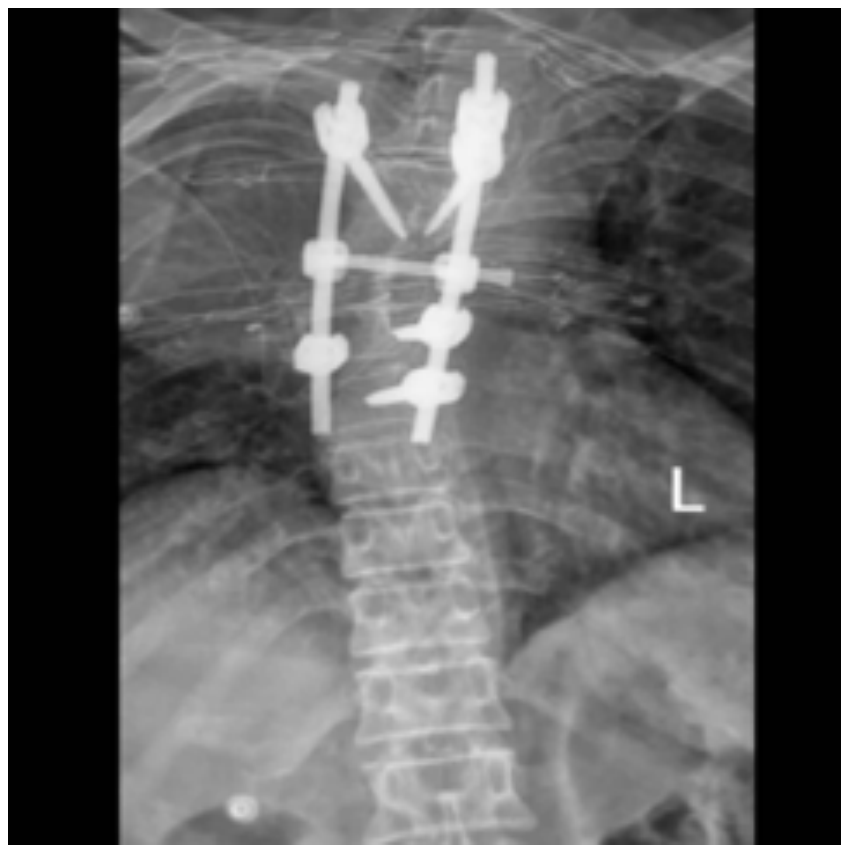

Figure 2. The postoperative anterior-posterior radiography showing a long posterior fusion using the pedicle screw from T3 to T7 levels

Central posterior vertebral body erosion and wedging, pedicle erosion, foraminal enlargement, and kyphosis are included in classic radiologic findings. Angular deformities (typically less than six levels of vertebrae), fractures of vertebrae, and dislocations are among the consequences of progressive vertebral body erosion [11]. Since the spinal canal is widened, neurologic deficit rarely occurs. Furthermore, due to the adverse effects of spinal cord compression, there is a chance of paraparesis or pain [13]. Spinal abnormalities grow and evolve into a condition known as "modulation" as the disease progresses. Surgical intervention is performed on patients with spinal deformities or symptomatic patients due to spinal cord compression and mediastinal structures. Early posterior fixation and fusion are required for the reduction and prevention of severe deformities and complications [11].

\section{Conclusion}

Neurofibromatosis type 1 manifests as dura weakness and results in focal enlargement, which is termed dural ectasia. Despite the fact that dural ectasia is an uncommon and benign condition, operation is often required for symptomatic, developing lesions.

\section{Ethical Considerations}

Compliance with ethical guidelines

Written informed consent was obtained from the patient.

\section{Funding}

This research did not receive any grant from funding agencies in the public, commercial, or non-profit sectors.

\section{Authors' contributions}

Conception, design, drafting the article: Mahsa Pakseresht Mogharab; Data collection: Mahsa Pakseresht Mogharab, Amin Naseri; Critically revising the article: Amin Naseri, Mahsa Pakseresht Mogharab; Reviewing submitted version of the manuscript, data analysis, and interpretation, approving the final version of the manuscript: All authors.

\section{Conflict of interest}

The authors declared no conflict of interest. 


\section{References}

[1] Friedman JM. Epidemiology of neurofibromatosis type 1. American Journal of Medical Genetics. 1999; 89(1):1-6. [DOI:10.1002/(SICI)1096-8628(19990326)89:1<1::AIDAJMG3>3.0.CO;2-8] [PMID]

[2] Wallace MR, Marchuk DA, Andersen LB, Letcher R, Odeh $\mathrm{HM}$, Saulino AM, et al. Type 1 neurofibromatosis gene: Identification of a large transcript disrupted in three NF1 patients. Science. 1990; 249(4965):181-6. [DOI:10.1126/science.2134734] [PMID]

[3] Li Y, O'Connell P, Breidenbach HH, Cawthon R, Stevens J, $\mathrm{Xu} \mathrm{G}$, et al. Genomic organization of the neurofibromatosis 1 gene (NF1). Genomics. 1995; 25(1):9-18. [DOI:10.1016/08887543(95)80104-T] [PMID]

[4] Sørensen SA, Mulvihill JJ, Nielsen A. Long-term follow-up of von Recklinghausen neurofibromatosis. Survival and malignant neoplasms. The New England Journal of Medicine. 1986; 314(16):1010-5. [DOI:10.1056/NEJM198604173141603] [PMID]

[5] Durrani AA, Crawford AH, Chouhdry SN, Saifuddin A, Morley TR. Modulation of spinal deformities in patients with neurofibromatosis type 1. Spine. 2000; 25(1):69-75. [DOI:10.1097/00007632-200001010-00013] [PMID]

[6] Akbarnia BA, Gabriel KR, Beckman E, Chalk D. Prevalence of scoliosis in neurofibromatosis. Spine. 1992; 17(Suppl 8): S244-8. [DOI:10.1097/00007632-199208001-00005] [PMID]

[7] Khong PL, Goh WHS, Wong VCN, Fung CW, Ooi GC. MR imaging of spinal tumors in children with neurofibromatosis 1. American Journal of Roentgenology. 2003; 180(2):413-7. [DOI:10.2214/ajr.180.2.1800413] [PMID]

[8] Tsirikos AI, Saifuddin A, Noordeen MH. Spinal deformity in neurofibromatosis type-1: Diagnosis and treatment. European Spine Journal. 2005; 14(5):427-39. [DOI:10.1007/s00586004-0829-7] [PMID] [PMCID]

[9] Ahn NU, Sponseller PD, Ahn UM, Nallamshetty L, Rose PS, Buchowski JM, et al. Dural ectasia in the Marfan Syndrome: MR and CT findings and criteria. Genetics in Medicine. 2000; 2(3):173-9. [DOI:10.1097/00125817-200005000-00003] [PMID]

[10] Toyoda K, Taguchi T, Kaneko K, Kato Y, Imajo Y, Imagama T. High-grade L5 spondylolisthesis associated with dural ectasia in neurofibromatosis. Journal of Orthopaedic Science. 2005; 10(2):233-6. [DOI:10.1007/s00776-004-0869-1] [PMID]

[11] Derdabi I, Jouadi HE, Edderai M. Dural ectasia: A manifestation of type 1 neurofibromatosis. Pan African Medical Journal. 2018; 31:226. [DOI:10.11604/pamj.2018.31.226.9797] [PMID] [PMCID]

[12] Jeong JW, Park KY, Yoon SM, Choe DW, Kim CH, Lee JC. A large intrathoracic meningocele in a patient with neurofibromatosis-1. The Korean Journal of Internal Medicine. 2010; 25(2):221-3 [DOI:10.3904/kjim.2010.25.2.221] [PMID] [PMCID]

[13] de Kleuver M, van Jonbergen JPW, Langeloo DD. Asymptomatic massive dural ectasia associated with neurofibromatosis type 1 threatening spinal column support: Treatment by anterior vascularized fibular graft. Journal of Spinal Disorders \& Techniques. 2004; 17(6):539-42. [DOI:10.1097/01. bsd.0000117544.88865.f0] [PMID] 